\title{
Elektronischer Geschäftsbericht 2015: ein nachhaltiger Schritt
}

\section{Anne-Geneviève Bütikofer}

lic. iur., Generalsekretärin der FMH

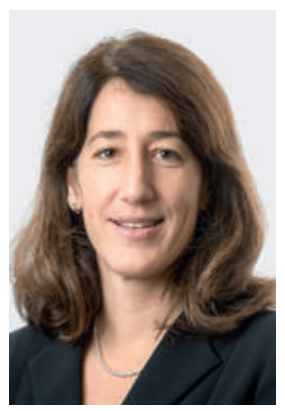

Ich freue mich, Ihnen planmässig den Geschäftsbericht 2015 der FMH präsentieren zu können. Dieser wird zwar zum gleichen Zeitpunkt wie in den Vorjahren veröffentlicht, im Gegensatz dazu aber dieses Mal nicht mehr in gedruckter Form bereitgestellt. Nicht nur sämtliche Mitglieder der FMH, sondern auch alle unsere Partner haben fortan auf diese ausschliesslich elektronische Version Zugriff.

Dabei stellt dieser als ePaper veröffentlichte Geschäftsbericht nur eine Übergangslösung dar, bis Sie ab Frühjahr 2017 über die FMH-Website auf unseren OnlineGeschäftsbericht in seiner endgültigen und interaktiven Form zugreifen können. In dieser nun unter dem Titel "Gesundheitsversorgung in der Schweiz - heute und morgen» veröffentlichten ePaper-Version 2015 finden Sie vertraute Rubriken wie den Rückblick auf die Tätigkeit der FMH, die Berichte des Zentralvorstands und der Abteilungen des Generalsekretariats, die Berichte über die internationalen Mandate, einen Auszug aus der Ärztestatistik sowie die Jahresrechnung, die gemäss den gesetzlichen Anforderungen für die Rechnungslegung nun auch einen Lagebericht umfasst. Zu guter Letzt auch das Podiumsgespräch, das auszugsweise in dieser SÄZ-Ausgabe (S. 494) enthalten ist und für welches Nationalrätin Ruth Humbel Näf, Medbase-CEO Marcel Napierala und FMH-Präsident Jürg Schlup in einer packenden Diskussion unter dem Motto des diesjährigen Geschäftsberichts am selben Tisch sassen.

Das Podiumsgespräch des diesjährigen Geschäftsberichts ist ein packender Austausch zum Thema "Gesundheitsversorgung in der Schweiz - heute und morgen».

Auch wenn der Entscheid zum Verzicht auf die gedruckte Version des Geschäftsberichts vor allem ökonomisch motiviert war, ist er auch ein Zeichen unsererseits, zum sozialen Verantwortungsbewusstsein und zur Nachhaltigkeit beizutragen. Schliesslich wenden die FMH als verantwortungsvoller Verband und ihr Generalsekretariat die Grundsätze der Corporate Social Responsibility (CSR) an, welche die gesellschaftlichen, ökologischen und wirtschaftlichen Auswirkungen unserer Tätigkeit berücksichtigen. Wenn man die Etappen der finanziellen Umstrukturierung, welche die FMH zuletzt durchlaufen hat, auf reine Massnahmen zur Budgetstabilisierung reduziert, verkennt man das Ausmass der Veränderung und Entwicklung, denen sich die FMH verschrieben hat. Unsere Vorgehensweise zielt vielmehr auf nachhaltiges und effizientes Handeln ab, welches im Einklang mit unseren Werten steht.

Selbstverständlich stellt der Wegfall des gedruckten Geschäftsberichts nicht die einzige Massnahme gemäss der CSR dar, die in den vergangenen Jahren oder

Der Verzicht auf die gedruckte Version

des Geschäftsberichts ist auch ein Beitrag zum sozialen Verantwortungsbewusstsein und zur Nachhaltigkeit.

im Rahmen der Budgetstabilisierung ergriffen wurde. Im Allgemeinen betrifft die CSR eine Vielzahl an Themen, die wir bei unserer Geschäftstätigkeit und in der Zusammenarbeit mit unseren Mitgliedern, Partnern und Mitarbeitenden berücksichtigen. Hier liessen sich zum einen unter anderem die Stärkung der Geschäftsführung, die Formalisierung der Arbeitsbedingungen sowie das betriebliche Gesundheitsmanagement für das Generalsekretariat nennen, zum anderen auch die Einführung eines Risiko- und Datenschutzmanagements, die Wahrung der Interessen unserer Mitglieder mit Hilfe der Neuausrichtung unseres Dienstleistungsangebots, die Überarbeitung unserer Grundsätze der Finanzverwaltung und die Transparenz nennen.

Zahlreiche Anstrengungen können umgesetzt werden oder sind noch umzusetzen, um unsere Abläufe zu optimieren und uns dauerhaft für Nachhaltigkeit einzusetzen. Dadurch können wir weiterhin dazu beitragen, uns den Herausforderungen zu stellen, vor denen unsere Gesellschaft steht.

Lesen Sie unseren Geschäftsbericht in elektronischer Form und nehmen Sie die Schlüsselereignisse des Jahres 2015 zur Kenntnis - damit leisten Sie auch einen Beitrag zu den vorhin erwähnten Anstrengungen. Ich wünsche Ihnen eine anregende, verantwortungsvolle Lektüre. 